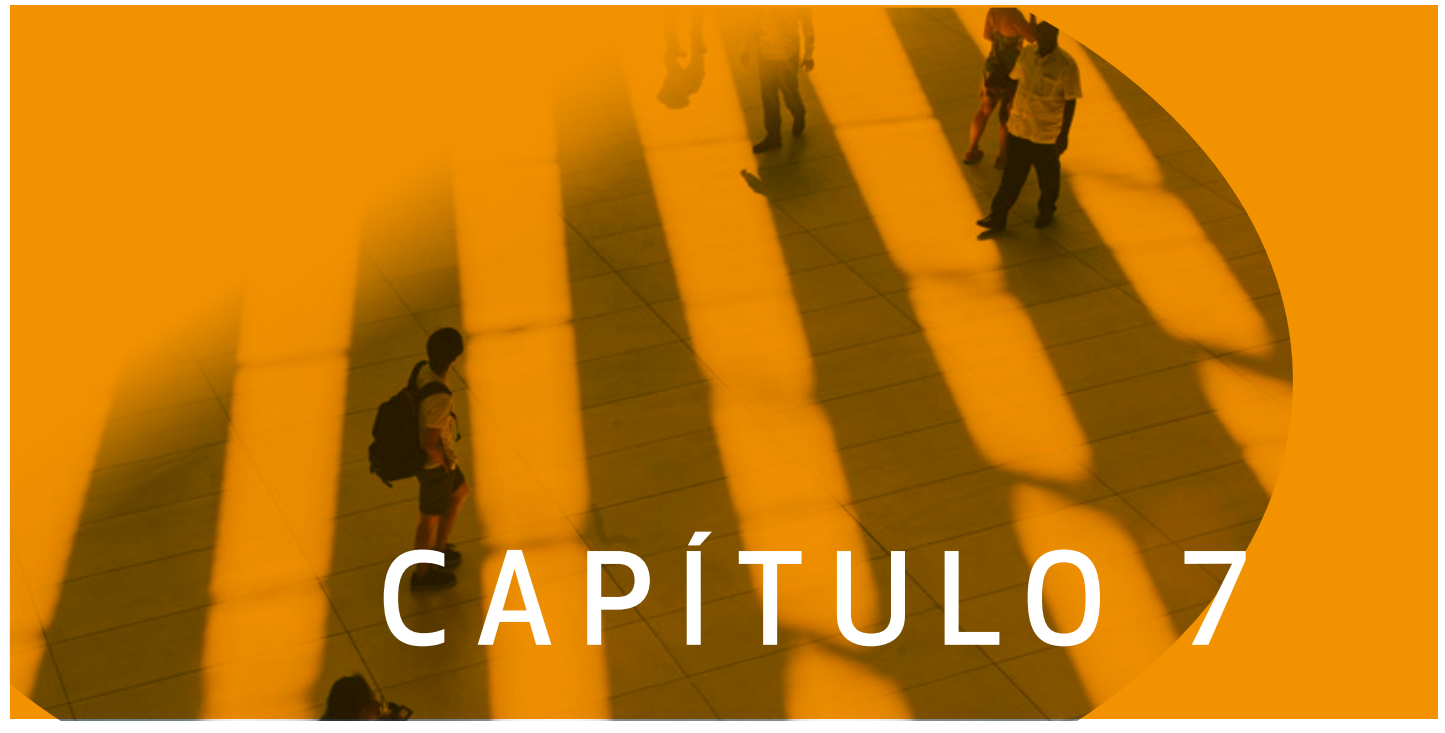





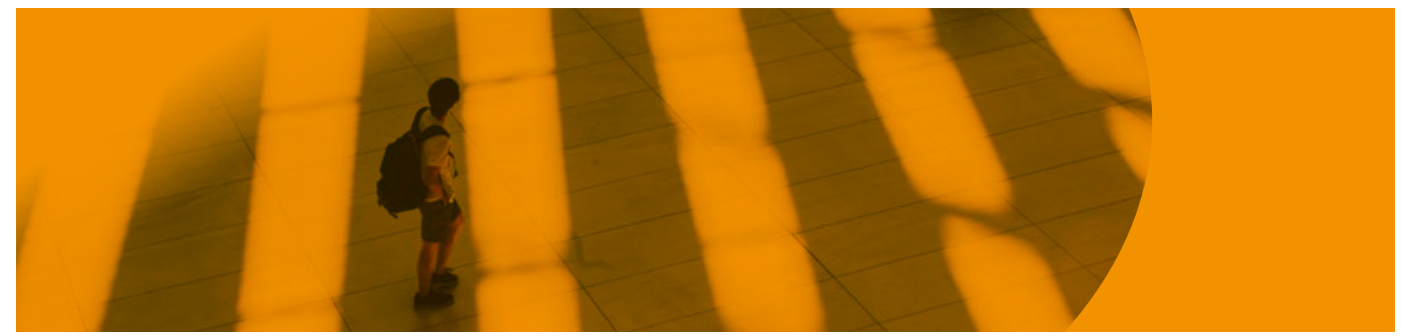

\section{La contabilidad social en la organización del siglo xxI}

\section{Julio Cesar Montoya Rendón}

Magister en Administración, Especialista en Pedagogía para el Desarrollo del Aprendizaje Autónomo, Administrador de Empresas, Investigador del grupo ILAMA. Universidad Nacional Abierta y a Distancia - UNAD

ORCID: https://orcid.org/0000-0003-1170-9296

Correo electrónico: julio.montoya@unad.edu.co

\section{Martha Lucía Fuertes Díaz}

Doctora en Administración Gerencial (C), Universidad Benito Juárez, México. Magíster en Administración con énfasis en Finanzas Corporativas, Universidad ICESI, Colombia. Especialista en Finanzas con concentración en Finanzas Avanzadas, Universidad ICESI, Colombia. Contadora Pública, Universidad Santiago de Cali, Colombia. Docente Universidad Nacional Abierta y a Distancia, UNAD, Colombia. Investigadora principal Grupo de Investigación ilama.

Correo electrónico: martha.fuertes@unad.edu.co

ORCID: http://orcid.org/0000-0001-8468-1867

Google Scholar: https://scholar.google.com.co/citations?user=IP5s- 


\section{Introducción}

Si bien el Estado es, en principio, el responsable del buen comportamiento de las variables sociales, este aspecto de la vida humana no es sólo responsabilidad de él. Las organizaciones también tienen responsabilidad frente a los múltiples y complejos hechos sociales donde es obvio que impactan y, a su vez, son permeadas por las condiciones sociales de sus entornos y por las políticas que enuncie el Estado para abordar el fenómeno social.

Es así que, desde mediados del siglo xx, la disciplina contable ha venido ampliando la visión economicista y empezó a considerar las dimensiones sociales que subyacen en la organización y en su contexto. Tendencia que ha tomado mayor fuerza en el siglo xxı, donde la organización, de manera más explícita, reconoce sus impactos sociales y ambientales e informa acerca de ellos. Al igual que lo hace con los tradicionales informes financieros. De esta manera, reconoce el contundente hecho de estar en una sociedad de organizaciones, donde son conformadas por personas y todos, a su vez, conforman el tejido social (Montoya et al., 2018).

A propósito de lo anterior, Mejía, Montes y Dávila (2011), enuncian algunos lineamientos que brindan una perspectiva más amplia de la contabilidad, a saber:

- Expresión en unidades monetarias y no monetarias.

- Cambios en el tiempo, cambios de objetos, hechos y personas y sus correspondientes relaciones.

- Existencia de una organización que cuenta con objetivos, propósitos, estructuras, jerarquías pertinentes y relaciones.

- Existencia de un sistema que requiere y genera información.

- La contabilidad aplica a todo tipo de organización.

- Ayuda al cumplimiento de los objetivos de la organización o del ente.

- Genera información histórica y predictiva.

- Procesa información para análisis de la realidad, no exclusivamente de la realidad financiera.

- Sirve a la toma de decisiones. 
- Informar de muchas situaciones más que el patrimonio.

- Los usuarios y generadores de información contable son los grupos de interés.

Por tanto, no es suficiente con presentar los estados financieros a los directivos e inversionistas de la organización para que éstos tomen decisiones. El ámbito es mayor y se hace necesario reportar información a todos los grupos de interés y sobre las temáticas que a cada grupo le compete. Es claro que la realidad es muy compleja y se deben considerar de manera sistémica y holística en todos sus componentes. Entre ellos, lo social, financiero, administrativo, económico, ambiental, y otros; como también, las múltiples interacciones que se dan entre los mencionados componentes.

La función social, que necesariamente tiene la organización, obliga a que informe periódicamente y sistemáticamente acerca de esta responsabilidad a todas sus audiencias, es decir, a sus grupos de interés. Por tanto, la organización debe medir la cuantía de sus impactos sociales y ambientales, tanto los que significan beneficios como aquellos que impactan negativamente y sobre las medidas de mitigación, sus costos y la solución propuesta.

Si bien la contabilidad nacional da cuenta de indicadores macro sociales, es necesario que la organización, además de la contabilidad financiera, dé cuenta de la contabilidad social dentro del ámbito de la disciplina contable y de su rigor científico. Esto da lugar a la presentación de informes sociales en los temas que son competencia de la organización.

La expresión de la contabilidad social debe ampliar el paradigma cuantitativo y de las unidades monetarias, en tal sentido, se pueden adoptar expresiones cualitativas y en unidades no monetarias. Se pasa así de la medición de variables a la definición de categorías en un ámbito más cualitativo. Ciertamente este abordaje social de la contabilidad requiere un marco teórico que le dé rigor científico y brinde los elementos para abordar la problemática de la contabilidad social. 


\section{Importancia de la contabilidad social}

La función social de la organización siempre ha estado implícita, en su origen está el satisfacer una necesidad de la sociedad, impacta socialmente con el empleo que brinda y genera riqueza para el país. En el siglo xxı, los hechos sociales que generan la organización o aquellos con los que es impactada, deben ser medidos, categorizados y comunicados por parte de la organización mediante los balances e informes que demanda el Estado y la sociedad. Esta información sirve para tomar decisiones y desarrollar una mejor gestión, es lo que se conoce como la contabilidad social.

Es así que la contabilidad social se puede entender como la ciencia o técnica que mide e interpreta los hechos sociales que generan o afectan a la organización. Incluye los costos y beneficios relacionados con los impactos sociales relevantes que son reportados mediante informes periódicos, los cuales son contrastados con los objetivos de la organización, a la vez que se convierten en insumos para tomar decisiones y realizar la planeación.

\section{Enfoques teóricos y temáticas de la contabilidad social}

Por la complejidad del contexto social donde están inmersas las organizaciones, necesariamente la contabilidad social debe tener un enfoque científico y multidisciplinar, con un fuerte soporte teórico dinámico que esté a tono con los constantes y rápidos cambios que sufren las organizaciones y sus contextos.

El modelo contable de lo social propone cómo se ordena y clasifica la información y su sistema, en el que se consideran los roles del emisor y usuario de dicha información. Se diferencian las cuentas, variables o categorías, según sean expresadas en unidades monetarias o no monetarias.

Como usuarios de esta información, se pueden considerar el Estado, los entes territoriales departamentales y municipales, consumidores, inversionistas y 
en general la sociedad que resulta afectada positiva o negativamente por la actuación de la organización.

Los hechos y fenómenos sociales, pertinentes a la organización, se pueden abordar desde diferentes teorías (Díaz, s.f.).

\section{Teoría neoclásica o de mercado}

La información se presenta en aquellos aspectos relacionados con el mercado donde el principal objetivo es que dicha información le sirva a los inversionistas, dado que lo comunicado puede tener influencia en el valor de la empresa o de sus acciones. Bajo el enfoque de la teoría del mercado, no se hace imperativo atender los requerimientos de la Responsabilidad Social Empresarial (RSE) dado que la máxima responsabilidad de la gerencia es con los objetivos de los inversionistas, es decir, con la rentabilidad. Se plantea que la máxima satisfacción social es posible bajo el ejercicio del libre mercado y la eficiencia con que las organizaciones atienden las necesidades de la sociedad. Este enfoque teórico con que se presenta la información es netamente económico y financiero, atiende sólo al grupo de interés de los inversionistas.

\section{Teoría social o interpretativa}

Considera que los entes u organizaciones están condicionadas por múltiples actores sociales -inversionistas, trabajadores, clientes, Estado, administradores y público en general- que son generadores y, a la vez, usuarios de la información que presentan. En este caso, cobra importancia el debate ético que pueden suscitar los balances financieros y los informes sociales que genera la organización, especialmente por sus impactos sociales. Esto en razón a que es importante y necesario para poder legitimar y justificar la naturaleza moral de la organización ante la sociedad en general. 


\section{Teoría crítica}

Este planteamiento es más radical, en el sentido que propone modelos de sociedad alternativos a los del mercado y, en consecuencia, propone también un rol diferente para la organización, la contabilidad y, por supuesto, para los contadores.

La postura crítica se opone contundentemente a la postura neoclásica del mercado, considera que las organizaciones deben de gravitar en torno a lo que determine la estructura social. En este punto es necesario anotar que, obviamente, la organización hace parte de la estructura social, teniendo en cuenta que se está en una sociedad de organizaciones.

La teoría crítica plantea que la contabilidad, más allá de mantener el statu quo del capital, debe involucrar explícitamente y contundentemente el componente social, lo que supone nuevos puntos de vista para presentar la información y, sobre todo, para interpretarla. No solo a la luz de rentabilidad, sino, teniendo en cuenta enfoques como la colaboración, la solidaridad y lo comunitario. Los promotores de la teoría crítica afirman que, si se ejerce la contabilidad bajo este enfoque, es una gran oportunidad de emancipación que brinda la oportunidad para desvanecer las grandes diferencias sociales.

\section{Temas que trata la contabilidad social}

Es importante tener en cuenta los siguientes aspectos no financieros de la organización, si bien se pueden valorar en unidades monetarias, es conveniente considerar mediciones o categorías no monetarias cuantitativas y cualitativas. Es una manera de tener una comprensión más exacta, sistémica y holística de estos aspectos tan importantes para la organización y su contexto social y económico.

\section{1). Ambientales:}

Es quizás el tema de mayor importancia que debe tratar la contabilidad social, dado el alarmante daño causado al medio ambiente por la actividad humana, especialmente por las empresas extractivas, de transformación y de transpor- 
te. Paralelo a este gran impacto nocivo para el ambiente, está la demanda de la sociedad por mitigar y reducir a cero este daño.

\section{2). Capital humano:}

Es importante que se presenten informes completos acerca del valor más importante, pues es realmente el que le da vida a la organización. Si no hay personas, no es posible concebir ésta. Información importante para la gestión de la organización, como también para alimentar los agregados económicos que contabiliza de manera macro el Estado.

\section{3). Ética:}

Aspecto importante e indispensable sobre el cual la organización está obligada a informar y evidencia la forma en que está dinamizando sus principios y valores. Se consideran aspectos como la defensa del consumidor, el aporte a la comunidad, solidaridad social, servicios a la sociedad, igualdad de oportunidades y de género, transparencia, entre otros. Algunas organizaciones desarrollan estas actividades a través de fundaciones.

\section{Objeto de estudio, metodología y principales problemas}

El avance científico de la contabilidad ha dibujado las fronteras del conocimiento contable más allá de la dimensión financiera y de su empírico uso reduccionista. Es así que incluye en su ámbito de estudio variables económicas, financieras, administrativas, ambientales y sociales, como también sus mutuas interacciones. Esto constituye una visión holística que es necesario, medir, relacionar, contrastar, describir e informar sistemática y metódicamente. De esta manera, involucra a todos los agentes que impactan y son impactados en el juego de la dinámica económica y social de las organizaciones.

En este contexto, el contador se convierte en un científico que observa un objeto de estudio complejo, a saber, la realidad organizacional y su contexto. 
Para realizar dicha observación cuenta con un corpus teórico que debe estar revisando y actualizando para que le permita definir operativamente variables y categorías para medirlas o describirlas, según sea el caso, e informar acerca de ellas y de sus mutuas interacciones de manera pertinente y objetiva.

Al tener la contabilidad social un objeto de estudio, una teoría y un método, se constituye en una disciplina que incluye diferentes campos, variables o categorías. Las cuales se pueden medir en unidades monetarias o no monetarias y, según sea el caso, pueden utilizar metodologías cuantitativas, cualitativas, mixtas e inclusive de acción y participación.

También se han definido dos niveles para la contabilidad social: macro y micro. Donde lo macro involucra las cuentas nacionales, los flujos de fondos, balanza de pagos, grandes agregados económicos, producción, ingreso, consumo, inversión, crecimiento, empleo, salud, educación, vivienda, entre otros, los cuales son abordadas desde la economía. Más lo micro que es abordado desde la contabilidad.

El mencionado enfoque macro de la contabilidad es alimentado por la suma de información sobre variables que se tratan desde el enfoque micro como responsabilidad social empresarial, capital humano, impactos ambientales, gestión del conocimiento, entre otros. De allí, la importancia de que, en la contabilidad de las organizaciones, se tengan en cuenta también las variables no financieras para poder medir cuál es el impacto que genera la actividad de la organización en las variables sociales. Esto se constituye en un valioso insumo para alimentar las cuentas nacionales, aclarando que la gestión de las variables sociales no son exclusiva competencia del Estado, son un compromiso de todas las organizaciones por el cual tienen que rendir cuentas (García, 2005).

Esta perspectiva obliga a que los investigadores contables, los organismos del Estado, los programas de contaduría, los centros de investigación, entre otros, continúen desarrollando la disciplina contable, identifiquen sus vacíos, descubran nuevos campos de aplicación, resuelvan nuevos problemas y, sobre 
todo, que la ciencia contable se constituya en una dinamizadora de desarrollo social para bien del ser humano y sus organizaciones.

La dinámica de la realidad, en permanente cambio, obliga a que las definiciones conceptuales siempre estén sometidos a prueba o validación, en el ejercicio de falsación que propone Popper (1997). Esto es necesario al considerar la contabilidad como una disciplina con estatus científico, reiterando que la contabilidad social cuenta con un cuerpo de teorías y conceptos, con su método empírico que permite obtener medidas y resultados de la realidad organizacional. Esto incluye los aspectos económicos y sociales, ya que también plantea cómo circula el dinero y los bienes en un contexto social y organizacional.

Cuando una disciplina, como la contabilidad social, cuenta con un conjunto de teorías y conceptos, puede formular hipótesis que se pueden probar o refutar, mediante la deducción, pero también puede construir nuevas teorías a partir de la inducción en una verdadera construcción epistemológica. Es una forma de abordar metodológicamente los problemas. En este sentido, no sólo se trata de emitir información contable que describa el estado de una organización o ente, se trata de qué interpretaciones se pueden hacer de esa información (Bunge, 2004). La ciencia contable cuenta con metodologías y herramientas para hacer interpretaciones y lecturas hermenéuticas de dicha información contable. Como también se pueden hacer comparaciones, bien sea de variables, cuando se está en el plano cuantitativo, o de categorías si se está en el plano cualitativo.

También se pueden hacer estudios transversales cuando se trata de "ver" la situación en un momento determinado o de estudios longitudinales cuando se trata de hacer una trazabilidad en un determinado lapso de tiempo. En este punto se puede ayudar de herramientas estadísticas, económicas, sociales, antropológicas, legales en un verdadero ejercicio inter, trans y multidisciplinario. Cuando se da este intercambio de saberes, necesariamente va surgiendo una comunidad de contadores investigadores, como en efecto ocurre en el presente.

Es necesario anotar que cuando la contabilidad trasciende el plano exclusivamente monetario, está cambiando de paradigma y ampliando su 
cuerpo de conocimiento, su objeto de estudio y la naturaleza de los problemas que aborda y sus estructuras (Kuhn, 2000). Pues son notorios los nuevos campos de aplicación de la contabilidad: forense, ambiental, social, entre otras. Es decir, al igual que otras ciencias, se ve enfrentado a cambios paradigmáticos y, porque no, a revoluciones metodológicas (Feyerabend, 1986)). Lo cual no sería posible sin la existencia de una comunidad científica contable como sistema social que intercambian saberes, comparten valores, continúan y a la vez transforman la tradición e investigan (Bunge, 2004). Lo anterior da lugar a un conflicto entre la práctica contable y la teoría, pero precisamente esto es lo que le da impulso a la disciplina contable.

\section{Definición, supuestos y objetivos de la contabilidad social}

El concepto de la contabilidad social no es nuevo, se viene mencionando desde el año 1963 (García, 2005). Desde entonces, ha tenido diferentes consideraciones apegadas a los contenidos sociales, destacando que la información contable, además de registrar hechos sociales, es de interés social. En el año 2000, Gray se compromete con una definición de la contabilidad social, en la cual se destaca que es la presentación de informes acerca de las mutuas interacciones y acciones de orden social, ambiental, humana, comunitarias, clientes, entre otros; así como las consecuencias de dichas acciones. Agrega que la contabilidad social además de la información financiera, debe considerar la información no financiera, información cuantificable, no cuantificable y descriptiva. Definición a la cual se adhiere Mathews, según afirma Quinche (2008).

En el año 1982, Gray y Perks presentan tres hipótesis claves en las que se fundamenta la contabilidad social:

1. Las empresas tienen responsabilidad social

2. Las empresas deben emitir informes sociales

3. Dichos informes sociales, deben ser elaborados por contadores según principios contables. (García, 2005). 
A continuación, se relacionan los supuestos básicos de la contabilidad que García (2005) toma y adapta de Mattessich para ser aplicados a la contabilidad social.

\section{Contabilidad de la entidad}

Existe un universo de las cuentas que son producidas, en el cual la contabilidad financiera, sólo es una pequeña parte. Se debe revisar el supuesto de la entidad que sólo considera la contabilidad financiera, lo que demanda un enfoque multidisciplinario necesario en la contabilidad social.

\section{Restricción a los hechos económicos}

En la contabilidad social también se tienen en cuenta y se registran los hechos no económicos, dando la importancia que tienen los hechos sociales.

\section{Limitación a los hechos económicos medidos en} términos financieros

Relacionar los hechos no financieros, es decir, los sociales en términos no monetarios y de forma cualitativa.

\section{Información limitada a la comunidad de las finanzas}

Los informes de la contabilidad no sólo van dirigidos a la comunidad financiera, el espectro de usuarios necesariamente se amplía a todos los grupos de interés.

De acuerdo a los anteriores supuestos, la contabilidad social debe contar con planes de cuentas sociales, codificaciones adecuadas y pertinentes que clasifique dichas cuentas, métodos y estudios de relación costo-beneficio, todo dentro de un modelo científico. Es necesario superar el reduccionismo a ultranza, donde la contabilidad social se asume independiente de las otras áreas de la organización y debe verse en el contexto integralmente con todas las áreas de la organización y su contexto. 


\section{Objetivos de la contabilidad social}

De las diferentes conceptualizaciones analizadas sobre la contabilidad social se pueden identificar los siguientes objetivos:

- Informar en forma transparente el comportamiento o impacto social de la organización mediante el balance social.

- Proporcionar información fidedigna a usuarios internos, externos, a la organización como insumo que permita la toma de decisiones sobre la gestión social que involucra a los grupos de interés.

- Aportar elementos claves para evaluar si las prácticas económicas, sociales, ambientales y éticas de la organización son consistentes con los intereses de los grupos interesados y con el desarrollo sustentable.

\section{Nuevos campos, alcance y contenido de la contabilidad social}

La contabilidad social parte de la consideración de variables macrosociales como la calidad de vida y los recursos naturales en sus dimensiones monetarias y no monetarias. Lo que permite hacer comparaciones a nivel de regiones, de países, de sectores económicos y de organizaciones.

Considerar variables como la pobreza, entre otras, esto con el fin de tener insumos para que se tomen decisiones a nivel de las organizaciones y de los organismos estatales con relación a la corrupción, la explotación de los trabajadores, políticas de desarrollo, bancarización y acceso al crédito, todo bajo el marco de las consideraciones éticas.

Es decir, que cuando se contabilizan las variables sociales, éstas se pueden controlar y así se coadyuva al desarrollo de la sociedad. Esta, en su conjunto, puede tener un bienestar dando cabida a términos como los de productividad social y rentabilidad social. 
Es lógico pensar que, si la organización recibe recursos de la sociedad, ésta informe que le está devolviendo a dicha sociedad, no sólo en valores económicos, sino de impacto social. Esto sin desconocer que hay una relación simbiótica entre la contabilidad financiera y la contabilidad social, relación que es necesario estudiar.

Necesariamente en la contabilidad social está inmerso el concepto de la Responsabilidad Social Empresarial (RSE). Si bien no hay normas claras frente a la presentación de informes de RSE, las empresas se ven obligadas a presentar completos informes al respecto, para los cuales tienen que llevar una serie de registros que les permitan consolidar la información a fin de que la organización dé cuenta de su gestión en el plano social y ambiental, lo que le genera una buena reputación ante sus audiencias.

Inevitablemente la empresa impacta socialmente y es impactada por sus entorno, pero es importante que se presente un informe al respecto y muestre sus resultados mediante indicadores que le permitan hacer un análisis integral de la responsabilidad social empresarial tanto dentro de la organización como afuera. Debe generar informes para la gestión de la organización puesto que le permite hacer el diagnóstico con respecto al cumplimiento de objetivos, metas y presupuestos de la Responsabilidad Social Empresarial.

A propósito de la Responsabilidad Social Empresarial, ésta debe estar enmarcada dentro de las políticas de la organización que dan los derroteros para planificar, controlar y definir la forma en que se han de distribuir los recursos de manera efectiva. Es aquí donde la contabilidad social ejerce un papel importante para coadyuvar a que la política se cumpla al igual que los objetivos, midiendo, registrando, controlando las consecuencias, costos, beneficios de las acciones económicas y sociales.

En un mundo globalizado, se incrementa la atención que se le debe dar a las demandas sociales y ambientales que hace la sociedad a las organizaciones. Es innegable el protagonismo que tiene la organización como agente que impacta el ambiente, la economía y la sociedad, lo que obliga a que informen 
sobre cómo contribuyen en estos aspectos, con la misma prioridad con que presentan los estados financieros de la organización.

Sin embargo, es necesario cuidarse de convertir la gestión social en otra unidad de negocio. Ha ocurrido que algunas organizaciones gastan más publicidad en divulgar sus acciones ambientales o sociales que los recursos que han invertido en dichas acciones. Lo que se constituye en una publicidad engañosa para limpiar la imagen de la compañía, es decir, la gestión social debe ser transparente y debe promover la filantropía y la equidad. Sin embargo, la filantropía no debe ser la única motivación, las consecuencias sociales de la actividad empresarial deben medirse objetivamente de tal manera que puedan presentar reportes que sean relevantes para los inversionistas, dado que les dará los insumos para gestionar prácticas ambientales sostenibles. También generarán hechos sociales responsables que se evidencian en mejores productos, procesos más inocuos para el ambiente y trato justo para las personas.

Es tal la importancia de la Responsabilidad Social Empresarial que en el año 2010 se emite la norma internacional voluntaria ISO 26000, la cual no aspira a ser un sistema de gestión como lo son las demás normas ISO. Pretende orientar a las organizaciones para que definan conceptos de base sobre la RSE y su relación con el desarrollo sostenible, comprometiéndose de manera voIuntaria al cumplimiento de la RSE, promoviendo e implementando que todas las áreas y niveles de la organización se identifiquen con estos conceptos. ISO 26000 se rige por siete principios, a saber:

1). Rendición de cuentas.

2). Transparencia.

3). Comportamiento ético.

4). Respeto por los intereses de los grupos de interés.

5). Respeto a la ley.

6). Respeto a la normativa internacional.

7). Respeto a los derechos humanos. 
Estos principios deben ser abordados de manera holística e integral, no se pueden tratar de manera independiente o decidir descartar alguno de ellos. Es oportuno decir que atender estos principios de manera interdependiente es ir en el sentido de las recomendaciones que da la OCDE (Codrington, Fiamingo, Romano; 2012)

Cuando la organización implementa ISO 26000, está integrando y comprometiendo a todas las áreas y estamentos con la RSE. En tal sentido, debe captar las iniciativas voluntarias, estudiarlas, formalizarlas e incorporarlas a los planes de acción y a los presupuestos, asignarles responsables, hacerles seguimientos, registrarlas e informarlas de manera transparente. Es en este proceso donde juega papel importante la contabilidad social y por supuesto el contador.

\section{Conclusiones}

Las disciplinas, por razones prácticas, han delimitado muy bien sus objetos de estudio de una manera reduccionista, sin embargo, es justo reconocer que esto ha permitido el avance de la ciencia. Sin embargo, la realidad demanda que se le aborde de una manera holística y reclama que las disciplinas trabajen colaborativamente para abordar sus problemas de manera integral.

En el caso de la contabilidad, los estadísticos y los economistas han venido trabajando los aspectos macroeconómicos y macrosociales por un lado; y los contadores han estado circunscritos a gestionar la información financiera de las organizaciones por el otro, sin que los unos aprovechen la información de los otros. Pero esto ha cambiado, en el siglo xxı todos los grupos de interés que gravitan en torno a la organización demandan información acerca de su gestión, por tanto, los contadores deben de interesarse en otras disciplinas como la economía, sociología, estadística, antropología, ecología para emitir los informes y balances de la organización, o trabajar en equipo con estos profesionales para generar una contabilidad total. 
De esta manera, el contador podrá hacer una completa cartografía de los hechos económicos (Hauque y Di Russo, 2012) y sociales que surgen de la actividad de la organización y de su relación con el contexto. Así, el contador toma protagonismo y genera información que le es útil a todos los grupos de interés y a quienes toman decisiones tanto al interior como al exterior de la organización.

\section{Referencias}

Bunge, M. (2004). La investigación científica. Su estrategia y su filosofía. Mexico: Siglo XXI Editores.

Codrington, A., Fiamingo, M. y Romano, J. (2012). Viabilidad y aplicación de la contabilidad social en empresas argentinas. UADE. Recuperado de: https:// repositorio.uade.edu.ar/xmlui/bitstream/handle/123456789/2487/Codrington. pdf?sequence $=1$

Díaz, M. (s.f.) La contabilidad social. Origen y paradigmas. Revista de investigación UNMSM. Recuperado de: http://revistasinvestigacion.unmsm.edu.pe/index.php/ quipu/article/viewFile/5513/4749

Feyerabend, P. (1986). Tratado contra el método. Madrid, España: Editorial Tecnos.

García Fronti, L. (2005). Fundamentación teórica de la contabilidad social, con énfasis en las cuestiones micro, Buenos Aires, Argentina: Universidad de Buenos Aires. Facultad de Ciencias Económicas. Recuperado de: http://bibliotecadigital.econ.uba. ar/download/tesis/1501-1177_GarciaFrontil.pdf

García, I. (2012). Problemas de la Contabilidad social y ambiental: Algunas reflexiones sobre la necesidad de un abordaje interdisciplinario. Contaduría Universidad de Antioquia, 60, 209-218. Recuperado de: https://aprendeenlinea.udea.edu.co/ revistas/index.php/cont/article/viewFile/14690/12843 
Hauque, S. y Russo de Hauque, L. (2012). Contabilidad social: eje integrador de las ramas contables. Escritos contables y de administración, 3(2), 75-114. Recuperado de: https://dialnet.unirioja.es/descarga/articulo/5252245.pdf

Kuhn, T. (2000). El camino desde la estructura. Barcelona: Paidos.

Machado, M. A. (2004) Dimensiones de la contabilidad social. Revista internacional Legis de contabilidad y auditoría, 17, 173-218. Recuperado de: http://legal. legis.com.co/document?obra=rcontador\&document=rcontador_7680752a7d8c404ce0430a010151404c

Mejía, E., Montes, C. y Dávila, G. (2011). Introducción a la propuesta contable de GarcíaCastlla. Cuadernos de contabilidad, 12 (30), 127-164. Recuperado de: http://www. scielo.org.co/pdf/cuco/v12n30/v12n30a06.pdf

Montoya, J. (2018). El aporte del conocimiento contable en la Nueva Gestión Pública. In: W. Sanchez, R. Giraldo, M. Caballero, L. Nieto, J. Sánchez, G. De la Cruz, J. Montoya, N. Agudelo, M. Díaz, G. Hernandez, K. Ortiz, H. Hurtado, M. Chica and A. Arias, ed., La nueva gestión pública frente a los desafíos de Colombia en el siglo XXI. Cali, Colombia: 1st ed. Universidad Libre Seccional Cali, p.30.

Popper, K. (1997). El cuerpo y la mente. Barcelona, España: Ediciones Paidos. I.C.E. de la Universidad Autónoma de Barcelona.

Quinche, F.L. (2008). Una evaluación crítica de la contabilidad ambiental empresarial. Revista Facultad de Ciencias Económicas: Investigación y Reflexión, 16(1), 197216. Recuperado de: http://www.scielo.org.co/scielo.php?script=sci_arttext\&pi$d=S 0121-68052008000100014 \& \operatorname{lng}=$ en\&tlng=es. 
